Ю. ЛИЛЛЕ, А. МЯННИК, А. ЯГОМЯГИ,

H. САМЕЛЬ, Т. САКС

\title{
О ПРЕПАРАТИВНОМ БИОСИНТЕЗЕ ПРОСТАГЛАНДИНОВ ГРУПП $E$ И $F$ С ИСПОЛЬЗОВАНИЕМ СИНТЕТАЗЫ В ВИДЕ АЦЕТОН-ПЕНТАНОВОГО ПОРОШКА
}

3а полтора десятилетия со времени выяснения образования простагландина $E_{2}\left(П \Gamma E_{2}\right)$ при биохимической окислительной циклизации арахидоновой кислоты $\left[{ }^{1,2}\right]$ найдено 8 возможностей метаболизма арахидоновой и других т. н. эссенциальных жирных кислот с участием целого ряда энзимов [3]. Последние локализованы преимущественно в тех или иных органах животных и человека. В настоящее время осуществляется препаративный синтез, приводящий к образованию ПГ $E$, и начаты работы по реализации других возможностей, в том числе получения некоторых стабильных ПГ групп $D$ и $F$, а также нестабильных промежуточных эндоперекисей ПГ групп $G$ и $H$.

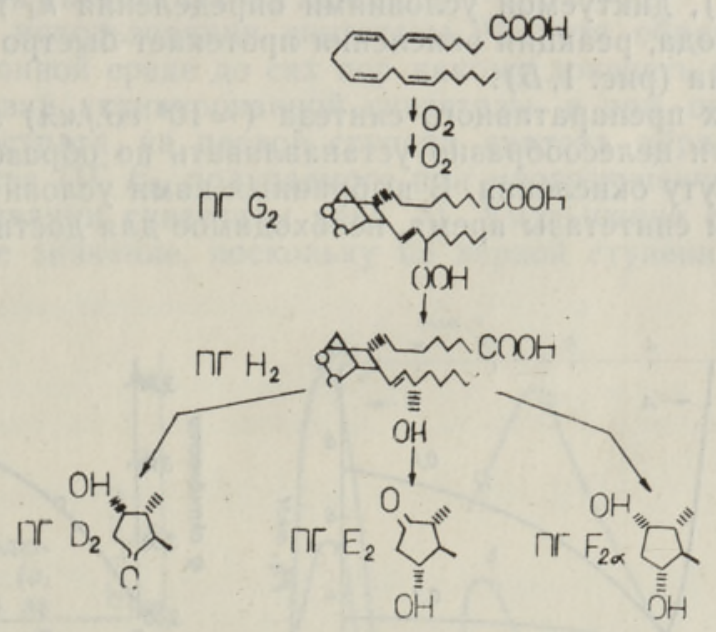

Биохимические методы синтеза ПГ - относительно нестабильных и оптически активных соединений - имеют в определенных условиях преимущества перед химическим синтезом, поскольку они просты и образование побочных продуктов при них минимально.

Среди работ по препаративному получению ПГ $E$ наиболее известна [ $\left.{ }^{4}\right]$, где комплекс энзимов (синтетаза) выделяется из семенных пузырьков барана в виде нерастворимого ацетон-пентанового порошка. Этим достигается многократное использование синтетазы, позволяющее

1 ENSV TA Toimetised. K 31979 
увеличивать выход продукта в 3 раза, по сравнению с выходом при применении гомогената ткани.

В данной статье изложены результаты первого этапа работ по препаративному получению ПГ биохимическим методом.

При осуществлении синтеза ПГ наиболее важными вопросами являются использование активности синтетазы и кинетика образования ПГ.

Активность синтетазы. Количество продукта $(P)$, полученного с некоторого количества энзима $(E)$, определяется уравнениями $\left[{ }^{5}\right]$ :

$$
\begin{aligned}
& {[P]_{t}=![E]_{0} \frac{k_{2}}{k_{i}}\left(1-e^{-k_{i} t}\right),} \\
& {[P]_{\infty}=[E]_{0} \frac{k_{2}}{k_{i}}=\frac{v_{0}}{k_{i}},}
\end{aligned}
$$

где $v_{0}$ - начальная активность синтетазы, $м \kappa M /$ мин (далее ед.); $k_{2}-$ каталитическая константа синтетазы, мин $-1 /$ ноль ме; $k_{i}-$ константа скорости инактивации синтетазы, мин $^{-1} ; t-$ время реакции, мин; $[P]$ и $[E]-$ соответствующие концентрации, мкM и мг/мл.

Константы $k_{2}$ и $k_{i}$ удобно определять по кривой поглощения кислорода с использованием кислородного электрода $\left[{ }^{5-7}\right]$. Полученные значения, в зависимости от времени и качества сбора семенных пузырьков, способа выделения и активации синтетазы, а также условий определения (тип буфера, наличие кофакторов, $\mathrm{pH}$ и температура среды), находятся в пределах $20-100$ и $0,2-1,0$ соответственно. Отметим, что с увеличением значения $k_{2}$ для конкретной синтетазы, как•правило, увеличивается и значение $k_{i}$.

Максимальный выход продукта на единицу активности синтетазы определяется величиной $k_{i}$ (рис. 1). При низкой концентрации энзима $(\approx 100 е \partial . / \mu \Omega)$, диктуемой условиями определения $k_{i}$ по кривой поглощения кислорода, реакция окисления протекает быстро до полной инактивации энзима (рис. $1, \overline{\text { э) }}$.

В условиях препаративного синтеза $\left(\approx 10^{4}\right.$ eд./м $)$ значение начальной активности целесообразно устанавливать по образованию продукта в первую минуту окисления. В выбранных нами условиях предварительной активации синтетазы время, необходимое для достижения половины

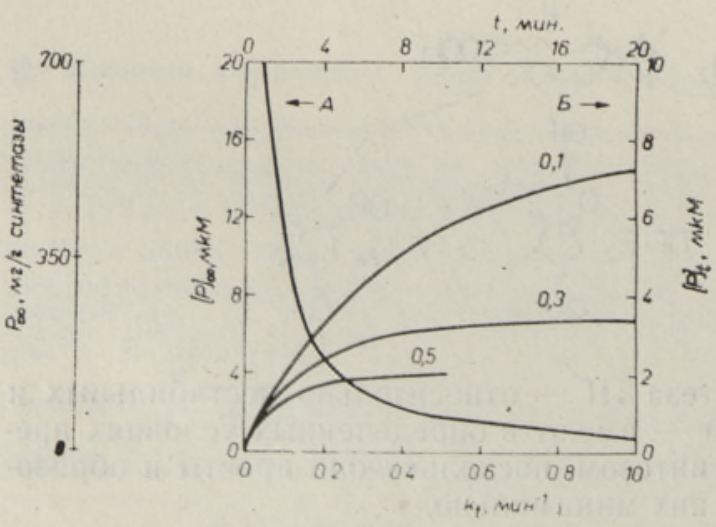

Рис. 1. Зависимость рассчитанных по уравнениям (1), (2) $[P]$ от $k_{i}$, Значения $P$ в ме/2 синтетазы рассчитаны при $k_{2}=100(A)$ и $[P]_{t}$ от $t$ при указанных на кривых значениях $k_{i}(D)$.

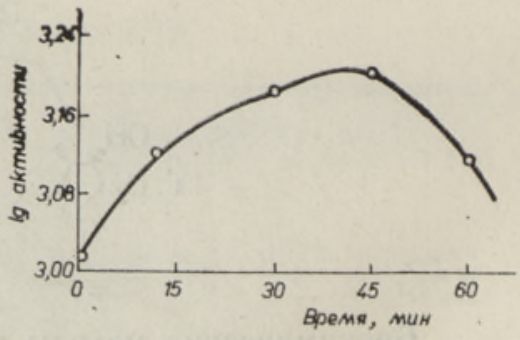

Рис. 2. Зависимость $v_{0}$ на первой ступени синтеза от времени активации. Значения $v_{0}$ рассчитаны из суммарного выхода ПГ $E_{2}$ на четырех последовательных ступенях синтеза с учетом распределения продукта по ступеням и времени (см. рис. 3). 
Рис. 3. Зависимость выхода ПГ $E_{2}$ от времени окисления в последовательных циклах работы синтетазы (неокрашенные точки слева; номера на кривых указывают очередность циклов) и инактивация синтетазы в циклах (темные точки справа). $\bigcirc$ и $\triangle(\square)$ - две партии арахидоновой кислоты. Условия работы активированной синтетазы: 75 мг/мл, ЭДТА-буфер 30 мM, субстрат (S) $3,4 \mu M$, глутатион 2 мM, гидрохинон $0,5 \boldsymbol{M M}$, температура $32^{\circ} \mathrm{C}$. Пунктиром обозначена временная зависимость количества ПГ $E_{2}$ на первой ступени синтеза при $[S]_{0}=4,2$ мM (вверху) и $2,5 \mu M$ (внизу).

максимальной активности, составляет $\approx 10$ мин (рис. 2 ). В случае активации оксибензеном это время равно 3,5 мин [7].

Кинетика образования ПГ $\boldsymbol{E}_{2}$. При высоких концентрациях энзима $(50-100$ мг/мл) скорость реакции образования ПГ $E_{2}$ в течение $10-$ 20 мин падает до нуля, но реакция возобновляется после замены жидкой реакционной среды свежей. Данное свойство ПГ-синтетазы является основой ступенчатого проведения синтеза ПГ $E_{2}$ при многократном использовании синтетазы [4], хотя образования ингибитора в реакционной среде до сих пор никому доказать не удалось.

В присутствии активированной синтетазы и при оптимальной концентрации субстрата на первой ступени синтеза образуется 65-80\% всего количества ПГ $E_{2}$, получаемого при многоступенчатом синтезе, до полной инактивации синтетазы (рис. 3). Уменьшение ступеней синтеза имеет большое значение, поскольку на первой ступени с активирован-

Рис. 4. Кривые элюирования ПГ $E_{2}(a$, б) и ПГ $F_{2 \alpha}(\boldsymbol{\theta})$ из колонок с силикагелем: $a$ - первая ступень очистки, элюент хлороформ-метанол; б - вторая ступень очистки, элюент гексан-этиловый эфир уксусной кислоты; в- вторая ступень очистки, элюент - тот же, что в 6 .

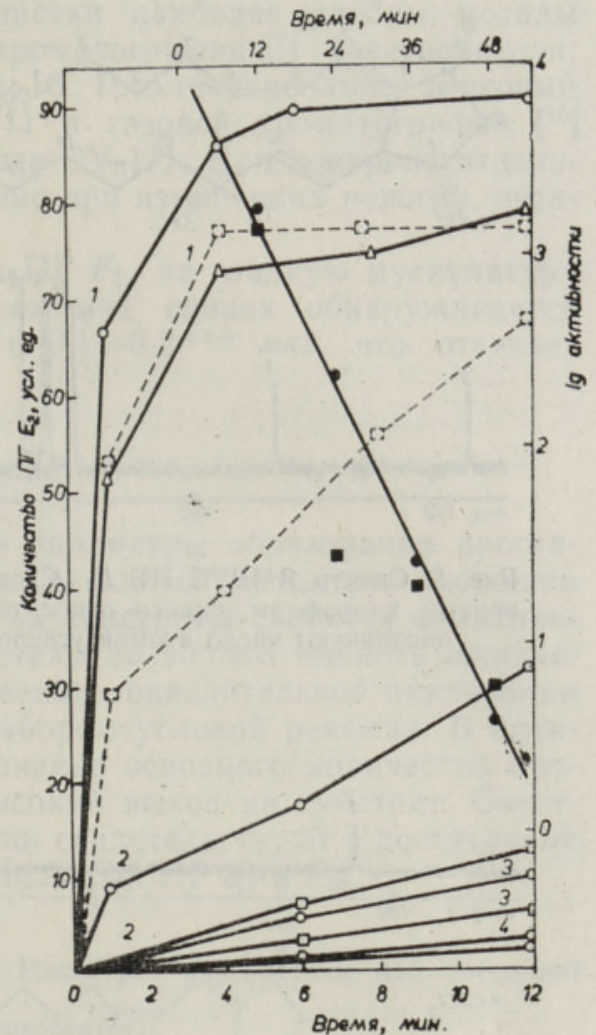



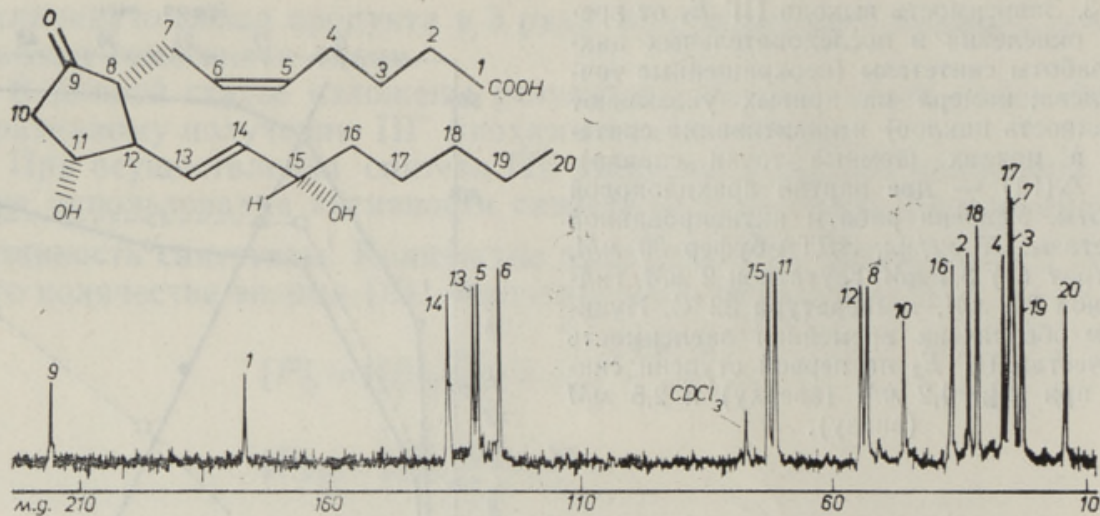

Рис. 5. Спектр ЯMP ${ }^{13} \mathrm{C}$ ПГ $E_{2}$. Спектрометр WH-90, растворитель дейтерированный хлороформ, сдвиги относительно тетраметилсилана. Цифры у пиков обозначают число атомов углерода, начиная с карбоксильной группы.

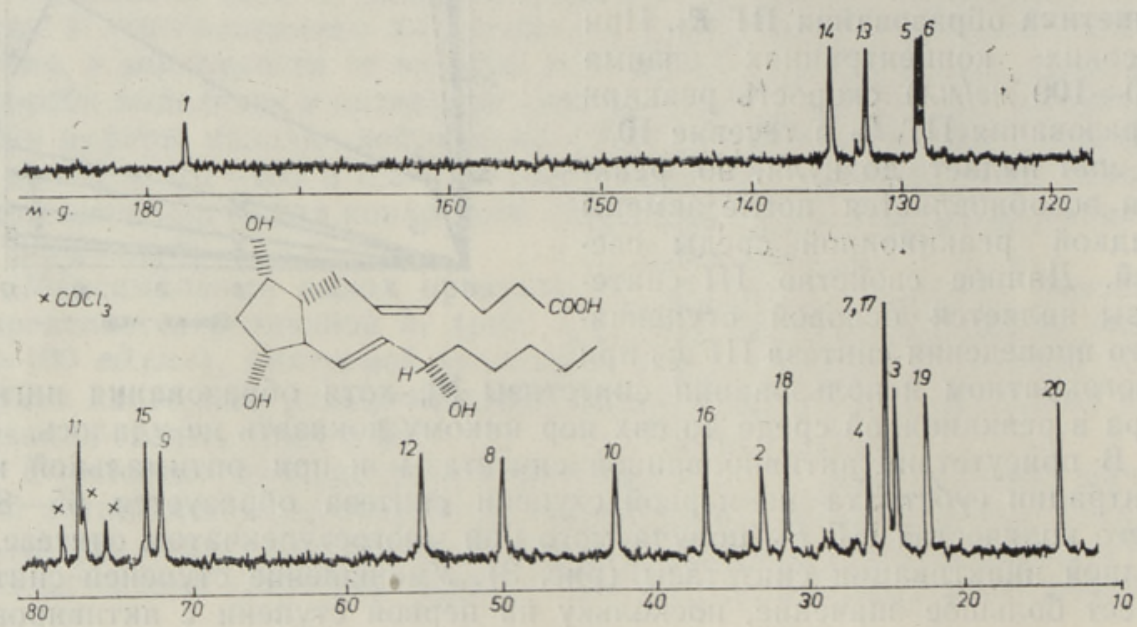

Рис. 6. Спектр ЯМР ${ }^{13} \mathrm{C}$ ПГ $F_{2 \alpha}$ (в дейтерированном метаноле).

ной синтетазой выход ПГ $E_{2}$ на арахидоновую кислоту составляет $60-70 \%$ (примерно в 2 раза выше по сравнению с выходом в десятиступенчатом процессе [ $\left.\left.{ }^{4}\right]\right)$.

Оптимальная чистота субстрата определяется экономическими критериями.

Синтез ПГ $\boldsymbol{F}_{2 \alpha}$. Увеличение масштаба опыта $\left.{ }^{8}\right]$ в $10^{3}-10^{4}$ раз и применение в качестве кофакторов соли двухвалентной меди и 2,3-димеркаптопропанола позволяют получить ПГ $F_{2 \alpha}$ в препаративных количествах.

Очистка и контроль качества продуктов. Для сокращения объема при . меняемых растворителей, времени их удаления и - главное - для получения чистых продуктов большую роль играет выбор оптимальных условий для вывода растворимых белков из сырой реакционной-смеси и для последующей очистки продукта. В последнем случае удовлетворительные результаты дает двукратное хроматографирование на силикагеле с применением известной системы растворителей [ $\left.{ }^{4}\right]$ (рис. 4$)$, при этом требуется стандартизация отдельных партий силикагеля. 
Для контроля за всем циклом очистки нанболее удобны методы щелочной деградации, тонкослойной хроматографии [] (растворители: бензол-диоксан-уксусная кислота $20: 10: 1$; детектирование: анисовый альдегид-этанол-конц. $\left.\mathrm{H}_{2} \mathrm{SO}_{4} \quad 1: 9: 1\right)$ и газовой хроматографии [ $\left.{ }^{10}\right]$ (триметилсилиловые производные в фазе OV-17). При контроле отдельных партий конечного продукта, особенно при изменениях режима, незаменим метод ЯMP ${ }^{13} \mathrm{C}\left[{ }^{11}\right]$ (рис. 5,6$) . *$

Действие синтезированных ПГ $E_{2}$ и ПГ $F_{2 \alpha}$ на гладкую мускулатуру изолированной матки белых крыс и морских свинок обнаруживается соответственно при дозах $0,1-1,0 * *$ и $0,1-0,3 * * *$ мк2, что отвечает стандартам.

\section{Заключение}

Истинные равновесные и кинетические параметры образования простагландинов в присутствии синтетазы в виде ацетон-пентанового порошка неизвестны. Однако кажущиеся значения константы скорости инактивации и каталитической константы синтетазы позволяют оценить ожидаемый максимальный выход продуктов реакции окислительной циклизации полиеновой кислоты, направляемый выбором условий реакции. В оптимальных условиях достигаются образование основного количества продукта на первой ступени синтеза и высокий выход на субстрат. Спектральные и биологические характеристики свидетельствуют о достаточной чистоте и высокой активности синтезированных ПГ $E_{2}$ и $F_{2 \alpha}$.

* Спектры снимались в секторе физики Института кибернетики АН Эстонской ССР Т. Пехком.

** Измерения О. Раявеэ (Тартуский госуниверситет).

*** Измерения Р. Борояна (Ереванский медицинский институт).

\section{Л И Т Е Р А Т У Р А}

1. Van Dorp, D. A., B e erthuis, R. K., Nugteren, D. H., Vonkeman, H. The biosynthesis of prostaglandins. - Biochim. Biophys. Acta, 1964, v. 90, p. $204-207$.

2. Berg st röm, S., D a n ie ls son, H., S a m u e ls s on, B. The enzymatic formation of prostaglandin $\mathrm{E}_{2}$ from arachidonic acid prostaglandins and related factors 32. - Biochim. Biophys. Acta, 1964, v. 90 , p. $207-210$.

3. Pace-Asciak, C. R. Oxidative biotransformation of arachidonic acid. Prostaglandins, 1977 , v. 13 , N 5, p. $8111-817$.

4. W a 11 a c h, P. D., D a n i e ls, G. E. Properties of a novel preparation of prostaglandin synthetase from seminal vesicles. - Biochim. Biophys. Acta, 1971, v. 231, p. $445-457$.

5. Лилле Ю., Смородин Е., М арвет Р. О кинетических свойствах простагландин-эндопероксид синтетазы. - Изв. АН ЭССР. Хим., 1979, т. 28 , № 2, c. $108-112$.

6. Rom e, H. L., L a n d s, W. E. M. Properties of a partially-purified of the prostaglandin-forming oxygenase from sheep vesicular gland. - Prostaglandins, 1975, v. 10, N 5, p. $813-824$.

7. S m it h, W. L., L a n d s, W. E. M. Oxygenation of polyunsaturated fatty acids during prostaglandin biosynthesis by sheep vesicular gland. - Biochemistry, 1972, v. 111,
N 17, p. 3276-3285.

8. L e e, R. E., L a nds, W. E. M. Cofactors in the biosynthesis of prostaglandins $F_{1}$ and $\mathrm{F}_{2}$. - Biochim. Biophys. Acta, 1972 , v. 260, p. $203-211$.

9. Kiefer, H. C., Johnson, C. R., Arora, K. L. Colorimetric indentification of prostaglandins in subnanomole amounts. - Analyt. Biochem., 1975, v. 68, p. $336-340$.

10. Rosello, J., T use11, J., Gelpi, E. Profiles of prostaglandins A, B, E and F (series I and II) obtained by gas chromatography with multiple-ion detection, - J. Chromatogr., 1977 , v. 130 , p. $65-76$. 
11. Cooper, G. F., Fried, J. Carbon-13 nuclear magnetic resonance spectra of prostaglandins and some prostaglandin analogs. - Proc. Nat. Acad. Sci. USA, 1973 , v. 70 , N 5, p. $1579-1584$.

Институт химии

Академии наук Эстонской ССР

Поступила в редакцию

23/XI 1978

O. LILLE, A. MANNIK, A. JAGOMAGI,

N. SAMEL, T. SAKS

\section{E- JA F-GRUPI PROSTAGLANDIINIDE PREPARATIIVNE BIOSUNTEES ATSETOON-PENTAANPULBRI ABIL}

Artiklis on esitatud järeldus, et sünteesi ulatuse ja teostuse määravad protsessi staatilised ja kineetilised parameetrid ning produktide eraldamise ja puhastamise meetodid.

ט. LILLE, A. MANNIK, A. JAGOMAGI,

N. SAMEL, T. SAKS

\section{ON PREPARATIVE SCALE BIOSYNTHESIS OF PROSTAGLANDINS E AND F GROUPS USING ACETONE-PENTANE POWDER SYNTHETASE}

Apparent values of inactivation rate constant and catalytic constant of acetone-pentane powder from sheep vesicular glands were used for the calculation of the biosynthesic capacity of PG-s. The oxygenative cyclization of polyene acids was directed toward E or $\mathrm{F}$ group PG-s. In optimal conditions the basic quantity of $\mathrm{PGE}_{2}$ was formed in the first cycle of synthesis, resulting in yields based on arachidonic acids, 3 -fold higher than were hitherto possible using multicycle process. The ${ }^{13} \mathrm{C}$ NMR spectra of products and the data of action on smooth muscle are given. 